\title{
Baker Alan R.H, Black Iain S. et Butlin Robin A., 130 Years of Historical Geography at Cambridge 1888-2018
}

Research Series, RGS with IBG, London 2019, 171 p.

\section{Philippe Boulanger}

\section{CpenEdition}

\section{Journals}

Édition électronique

URL : https://journals.openedition.org/geohist/1453

DOI : 10.4000/geohist. 1453

ISSN : 2264-2617

Éditeur

Association française de la Revue de géographie historique

Référence électronique

Philippe Boulanger, «Baker Alan R.H, Black lain S. et Butlin Robin A., 130 Years of Historical Geography at Cambridge 1888-2018», Revue de géographie historique [En ligne], Comptes-rendus, mis en ligne le 20 novembre 2020, consulté le 19 septembre 2021. URL : http://journals.openedition.org/geohist/1453 DOI : https://doi.org/10.4000/geohist.1453

Ce document a été généré automatiquement le 19 septembre 2021.

\section{(i) $\$$}

Ce(tte) œuvre est mise à disposition selon les termes de la Licence Creative Commons Attribution Pas d'Utilisation Commerciale - Pas de Modification 4.0 International. 


\section{Baker Alan R.H, Black Iain S. et Butlin Robin A., 130 Years of Historical Geography at Cambridge 1888-2018}

Research Series, RGS with IBG, London 2019, 171 p.

\section{Philippe Boulanger}

\section{RÉFÉRENCE}

Baker Alan R.H, Black Iain S. et Butlin Robin A., 130 Years of Historical Geography at Cambridge 1888-2018, Research Series, RGS with IBG, London 2019, 171 p.

1 Alan Baker, Iain Black et Robin Butlin proposent un ouvrage peu commun sur le développement de l'enseignement et de la recherche de la géographie historique à Cambridge. Certains de ces prestigieux collèges comme, Trinity College et Emmanuel College, ont été des lieux de rayonnement essentiel en Grande-Bretagne. Des générations de chercheurs et d'enseignants y ont été formés et ont diffusé, à leur tour, leurs savoirs dans bien d'autres universités britanniques. Faut-il rappeler que la géographie anglaise se partageait traditionnellement en géographie physique, géographie humaine et géographie historique. Autant dire que celle-ci constitue une approche importante de la géographie anglaise qui fut notamment soutenue par la Royal Geographical Society à la fin du XIX siècle et renforcée lors du $6^{e}$ congrès international de géographie de Londres en 1895.

2 L'année 1888 marque véritablement le début de cet enseignement à Cambridge par la création du premier poste de maître assistant en géographie financé par la Royal Geographical Society. La géographie historique du sacré et celle des grandes civilisations, nous apprennent les auteurs, constituent les premières thématiques fortes qui se sont diversifiées par la suite. La géographie des explorations, de l'expansion coloniale, des Etats et des subdivisions administratives, des populations et de l'économie, des paysages et de la démographie, politique et sociale à différentes périodes, ont été des 
approches centrales au XXe siècle. L'ouvrage nous propose d'en suivre les mutations selon une démarche chronologique en 8 chapitres : les débuts 1888-1919, les premières années 1919-1928, la réforme 1928-1945, maturité et spécialisation 1945-1966, la renaissance 1966-1976, les améliorations 1976-2003, les nouvelles directions 2003-2018.

Des grands noms mondiaux de la géographie historique y ont développé des connaissances fondamentales tel H. C. Darby qui y enseigne de 1931-1945 et de 1966 à 1976 et publie la célèbre étude Domesday Englanden 7 volumes (1954-1977) et A New Historical Geography of England(1973. Sa méthodologie et ses conclusions sont toujours des références en histoire médiévale. Il est à noter que la géographie historique de la France y a été enseignée pendant plusieurs décennies, notamment par Alan Baker qui a aussi encadré des générations de jeunes chercheurs dans les archives départementales en France entre 1976 et 2003. Les paysages ruraux et urbains, les sociétés sportives et militaires entre 1871 et 1914, et biens d'autres thématiques sur la France, ont fait l'objet de thèses de doctorats et de multiples publications. Une revue de référence, Journal of Historical Geography, y est publiée, aujourd'hui en format numérique. Si la géographie historique dans certains collèges à Cambridge continue d'être enseignée, notamment par Philip Howell et Alice Reid en démographie, David Nally et Matthew Gandy en culture urbaine et environnementale, Bill Adams en écologie politique, la discipline a toutefois perdu en rayonnement comme dans toutes les universités européennes où elle s'était installée. En 2018-2019, seulement huit professeurs sur quarante (20\%) du département de géographie à Cambridge, adoptent une perspective historique dans leurs recherches, six d'entre eux développent une approche en géographie historique. Mais, comme l'écrivent les auteurs, la géographie historique est bien vivante avec des approches renouvelées qui caractérisent l'évolution de la géographie académique.

En citant tous les professeurs, leurs publications et les manifestations organisées en géographie historique, cet ouvrage se veut une mise en perspective rigoureuse et passionnante, sans équivalent outre-Manche. Ses auteurs restituent aussi ce rayonnement intellectuel à l'échelle du pays en guise de conclusion. Les appendices donnent à comprendre la profusion des réflexions et des manifestations à différentes périodes. Des cartes et des tableaux illustrent de manière précise toutes ces informations, notamment les principaux lieux des manifestations de géographie historique en Grande-Bretagne. Sans nul doute, 130 Years of Historical Geography at Cambridge 1888-2018 constitue une somme de connaissances à découvrir sur les tenants et sur les axes forts de développement de cette branche de la géographie à Cambridge. 